\title{
Stress Anisotropy Dependent Ferromagnetic Resonance Frequency in Ferromagnetic/Antiferromagnetic Bilayers
}

\author{
H. WANG ${ }^{a}$, J.H. RONG ${ }^{b, *}$, G.H. YUN ${ }^{b, c}$ AND L.B. BAO ${ }^{b}$ \\ ${ }^{a}$ College of Science, Inner Mongolia Agricultural University, Hohhot 010018, P.R. China \\ ${ }^{b}$ Inner Mongolia Key Lab of Nanoscience and Nanotechnology and School of Physical Science and Technology, \\ Inner Mongolia University, Hohhot 010021, P.R. China \\ ${ }^{c}$ College of Physics and Electronic Information, Inner Mongolia Normal University, Hohhot 010022, P.R. China
}

(Received January 18, 2019; revised version July 15, 2019; in final form July 29, 2019)

The effect of stress anisotropy on ferromagnetic resonance frequency is investigated in ferromagnetic/antiferromagnetic bilayers with exchange anisotropy and uniaxial magnetocrystalline anisotropy. The intensity and the direction of applied magnetic field dependence of resonant frequencies are studied for different stress anisotropies. It is shown that resonant frequency can be tunable by the intensity and the direction of the stress anisotropy. Moreover, the easy axis and hard axis can be changed significantly by taking into account stress anisotropy field and exchange anisotropy field. The jump phenomenon in the angular dependence of stress anisotropy has been explained by analyzing the magnetization reversal processes.

DOI: 10.12693/APhysPolA.136.405

PACS/topics: ferromagnetic resonance, exchange anisotropy, stress anisotropy

\section{Introduction}

Since the exchange bias between ferromagnetic (FM) and antiferromagnetic (AFM) bilayers was discovered by Meiklejohn and Bean in 1956 [1], this model system has remained a very active research area, due to its applications in spintronic devices $[2,3]$ such as magnetic random access memory [4-6]. These devices often exhibit high stress when they are deposited on flexible substrate, which could affect their static magnetic configuration and propagation of spin waves [7-11]. However, most of the investigations have focused on the interlayer coupling and exchange bias [12-18]. Reports on the stress anisotropy in literatures are more scarce [19, 20]. In fact, the application of stress on the magnetic material can change its magnetic properties and result in different magnetic induction for a given magnetic field at a different value of the applied stresses [21]. Its underlying physical phenomenon is still not completely clear. Ferromagnetic resonance (FMR) [22] is an excellent tool for quantitative determination of the interlayer exchange coupling for different kinds of magnetic structures [23-26], and can be used to better understand multilayer systems. Nevertheless, the dependence of FMR on the stress anisotropy in multi-layers is not completely clear.

In our previous works [27-29], we have investigated the dependence of spin-wave resonance frequency on the surface anisotropy and the interlayer exchange coupling by using the linear spin-wave approximation and Green's function method. The interlayer exchange coupling is more important for the higher energy modes.

*corresponding author; e-mail: jhrong502@163.com
The purpose of the present work is to investigate theoretically the effect of the in-plane stresses on FMR behavior in FM/AFM bilayers. The motivation is to show how to obtain high and controllable FMR frequency.

The paper is organized as follows. The analytic derivation is obtained in Sect. 2. Section 3 discusses the effect of the exchange anisotropy field, the intensity and the direction of the stress anisotropy field, the direction of applied magnetic field on FMR frequency in ferromagnetic/antiferromagnetic bilayers. Section 4 gives a summary and conclusion.

\section{Model and calculation procedure}

The system under research here are the coupled bilayers. The geometry and coordinate system employed in the study are shown in Fig. 1. The two films FM/AFM are assumed to lie in the $x-y$ plane, with the $z$ axis normal to the film plane. The exchange anisotropy along the $x$ axis is modeled in terms of an effective field $H_{E}$. As indicated in Fig. 1, $\theta$ and $\phi$ correspond to the polar and azimuthal angles of the magnetization. The direction of applied magnetic field lies on film plane making an angle $\phi_{H}$ with the $x$ axis. In this work, we only consider in-plane stress, directed at angle $\alpha$ with the $x$ axis. We assume the magnetostriction of the two films to be isotropic. With all these considerations, the total free energy per unit volume in $\mathrm{FM} / \mathrm{AFM}$ bilayers can be explicitly written as

$$
\begin{aligned}
& \frac{E}{M}=-H \sin \theta \cos \left(\phi_{H}-\phi\right)-H_{E} \sin \theta \cos \phi \\
& +\frac{H_{\text {Keff }}}{2} \sin ^{2} \theta+\frac{H_{A}}{2} \sin ^{2} \theta \sin ^{2} \phi \\
& \quad-\frac{3}{2} \frac{\lambda_{s} \sigma}{M} \sin ^{2} \theta \cos ^{2}(\alpha-\phi) .
\end{aligned}
$$


Here the first two terms of Eq. (1) represent the Zeeman energy and the interfacial exchange anisotropy energy, respectively. The third term is the effective anisotropy energy including the shape anisotropy and out-of-plane uniaxial magnetocrystalline anisotropy energy, and the fourth term is an in-plane uniaxial anisotropy energy, which is defined by $H_{A}=\frac{2 K_{u p}}{M}$ (with $K_{u p}$ as the anisotropy constant) and taken to be along $y$ axis. The last term describes in-plane stress anisotropy energy, $\sigma$ and $\lambda_{s}$ denote the stress and magnetostriction constant, respectively. For simplicity, the interaction of the antiferromagnetic layer with the applied magnetic field is assumed to be negligible. The equilibrium position of the magnetization can be obtained from the first derivatives condition $\partial E / \partial \theta=0$ and $\partial E / \partial \phi=0$. It can easily be seen that $\theta=\pi / 2$ is a solution of the equilibrium equation. The magnetization must lie on the film plane. Its direction will be determined from the following equation

$$
\begin{aligned}
& H \sin \left(\phi_{H}-\phi\right)= \\
& \quad H_{E} \sin \phi+\frac{H_{A}}{2} \sin 2 \phi+\frac{3}{2} H_{\sigma} \sin 2(\phi-\alpha),
\end{aligned}
$$

where $H_{\sigma}=\lambda_{s} \sigma / M$ is defined as the stress anisotropy field.

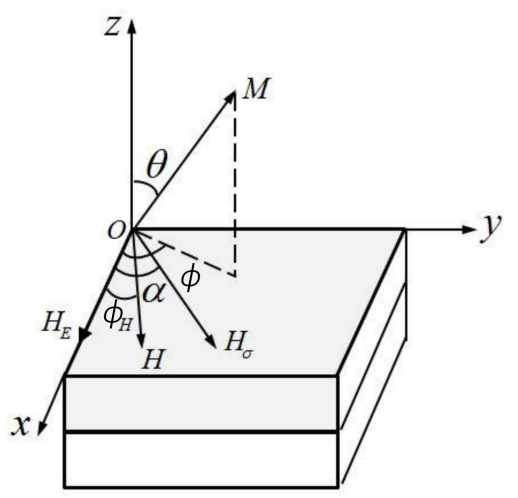

Fig. 1. Sketch of the theoretical model adopted for the numerical calculation.

The resonance condition can be derived using the Smit and Beljers relation [30]:

$$
\left(\frac{\omega}{\gamma}\right)^{2}=\frac{1}{M^{2} \sin ^{2} \theta}\left[\frac{\partial^{2} E}{\partial \theta^{2}} \frac{\partial^{2} E}{\partial \phi^{2}}-\left(\frac{\partial^{2} E}{\partial \theta \partial \phi}\right)^{2}\right] .
$$

Here $\omega$ denotes the angular frequency of microwave field, and $\gamma$ is the gyromagnetic ratio. An explicit expression derived from Eq. (3) has the form

$$
\begin{aligned}
& \left(\frac{\omega}{\gamma}\right)^{2}=\left[H \cos \left(\phi_{H}-\phi\right)-H_{K e f f}+H_{E} \cos \phi\right. \\
& \left.-H_{A} \sin ^{2} \phi+3 H_{\sigma} \cos ^{2}(\phi-\alpha)\right] \\
& \quad \times\left[H \cos \left(\phi_{H}-\phi\right)+H_{E} \cos \phi\right. \\
& \left.\quad+H_{A} \cos 2 \phi+3 H_{\sigma} \cos 2(\phi-\alpha)\right] .
\end{aligned}
$$

\section{Results and discussion}

Figure 2 illustrates how the direction of applied magnetic field affects the FMR frequency with different exchange and stress anisotropy fields yield the following values of the effective field $H_{\text {ueff }}=-10 \mathrm{kOe}$ and $H_{A}=0.005 \mathrm{kOe}$. Here, the applied magnetic field $H$ is equal to $1 \mathrm{kOe}$ in the following numerical calculation [12] and the direction of stress anisotropy field $\alpha$ is $90^{\circ}$. Let us look at the details. In case of the exchange anisotropy field is equal to zero (Fig. 2a, b), it is easily seen that the curves of the resonant frequency, in the absence of exchange anisotropy field, are twofold symmetry, and the corresponding easy and hard axes are along the directions with $\phi_{H}=0^{\circ}, 180^{\circ}$ (the [100] direction) and $\phi_{H}=90^{\circ}, 270^{\circ}$ (the [010] direction) in absence of the stress anisotropy, respectively. Whereas in presence of the stress anisotropy, the situation is reversed. It means that the stress anisotropy field can also alter the easy and the hard axis of the FM/AFM bilayers, which agrees with the results reported in Ref. [20]. Furthermore, the effect of stress anisotropy field on the symmetry is less pronounced. It still shows uniaxial anisotropy. When the exchange anisotropy field is increased (Fig. 2c), the original symmetry is destroyed. The easy axis is only along the positive direction of $x$ axis, and the corresponding resonant frequency is shifted to a higher value for $\phi_{H}=0^{\circ}$ or $\phi_{H}=360^{\circ}$ and to a lower value for $\phi_{H}=180^{\circ}$ (the hard axis direction) compared with Fig. 2a. It shows unidirectional anisotropy. With increase in exchange anisotropy field, the resonant frequency is shifted higher for $\phi_{H}<100^{\circ}$ and $\phi_{H}>258^{\circ}$. As the stress anisotropy field and the exchange anisotropy field are all included (Fig. 2d), the original symmetry is destroyed further. The secondary easy axis appears at $\phi_{H}=73^{\circ}$ and $\phi_{H}=287^{\circ}$ for $H_{\sigma}=0.03 \mathrm{kOe}$. Interestingly, for further increase in the stress anisotropy field, we found that resonant frequency value turns higher in both $45^{\circ}<\phi_{H}<135^{\circ}$ and $225^{\circ}<\phi_{H}<315^{\circ}$ regions. Obviously, in this condition, the in-plane stress anisotropy greatly influences the FMR frequency.

To further clarify the relations of both the magnetization angle $\phi$ and the direction of the applied magnetic field $\phi_{H}$, we have shown the difference between them in Fig. 3 for different exchange anisotropy and stress anisotropy fields. The corresponding parameters are chosen to be the same as in Fig. 2. When the exchange and stress anisotropies are absent (Fig. 3a), the deviation of the equilibrium direction of the magnetization from the direction of applied field is less pronounced. However, when the exchange anisotropy (Fig. 3b) or stress anisotropy (Fig. 3c) is taken into account, the difference increases, especially for stress anisotropy (it can reach 15 degrees for $H_{\sigma}=0.03 \mathrm{kOe}$ ). When the exchange anisotropy and stress anisotropy are all present (Fig. 3d), we found that difference increases in some regions $\left(\phi_{H}<90^{\circ}\right.$ and $\left.180^{\circ}<\phi_{H}<270^{\circ}\right)$.

The angle $\phi$ is determined by Eq. (2). 

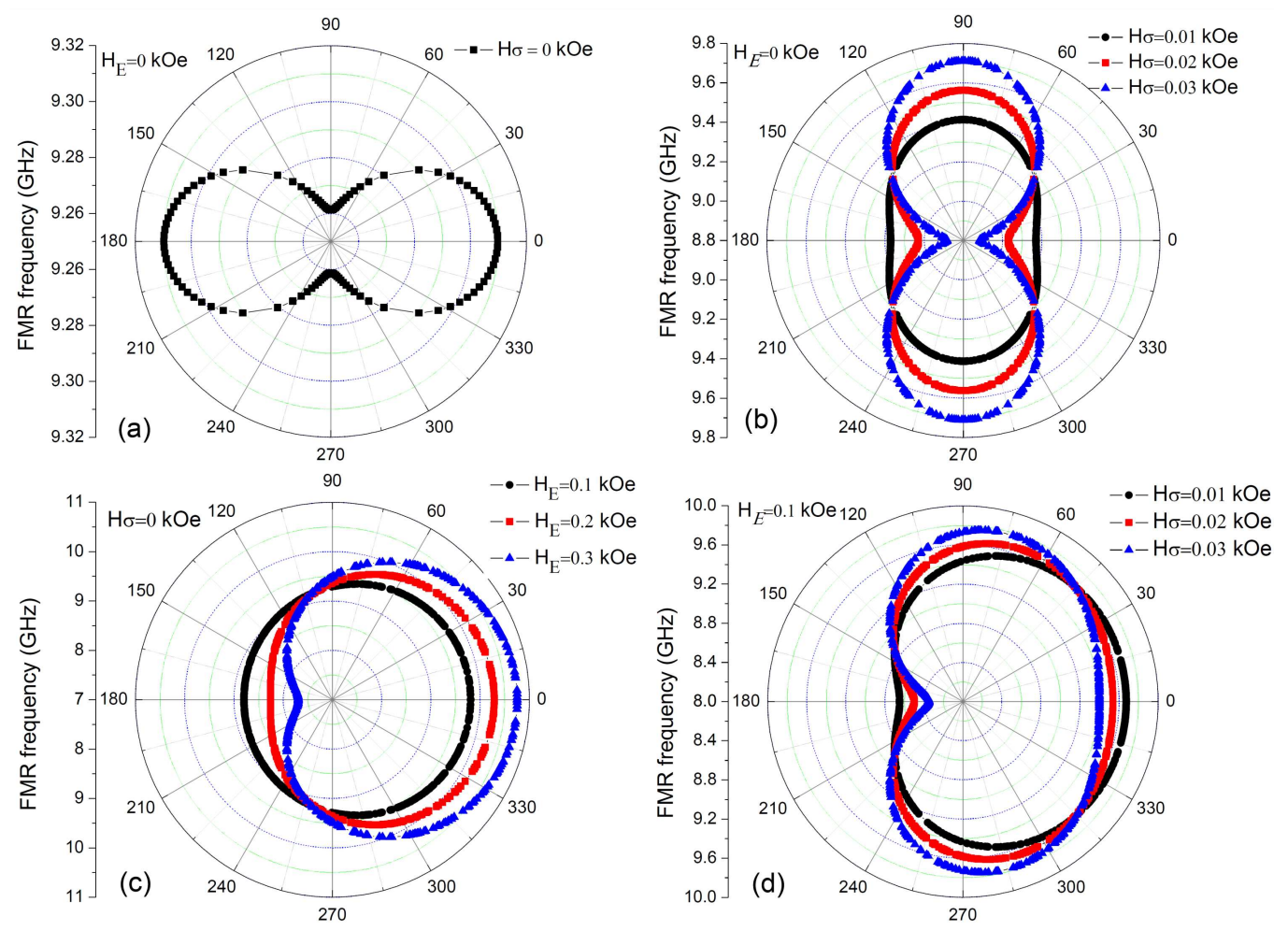

Fig. 2. Angular dependence of ferromagnetic resonance frequency with different stress anisotropy fields and exchange anisotropy fields.
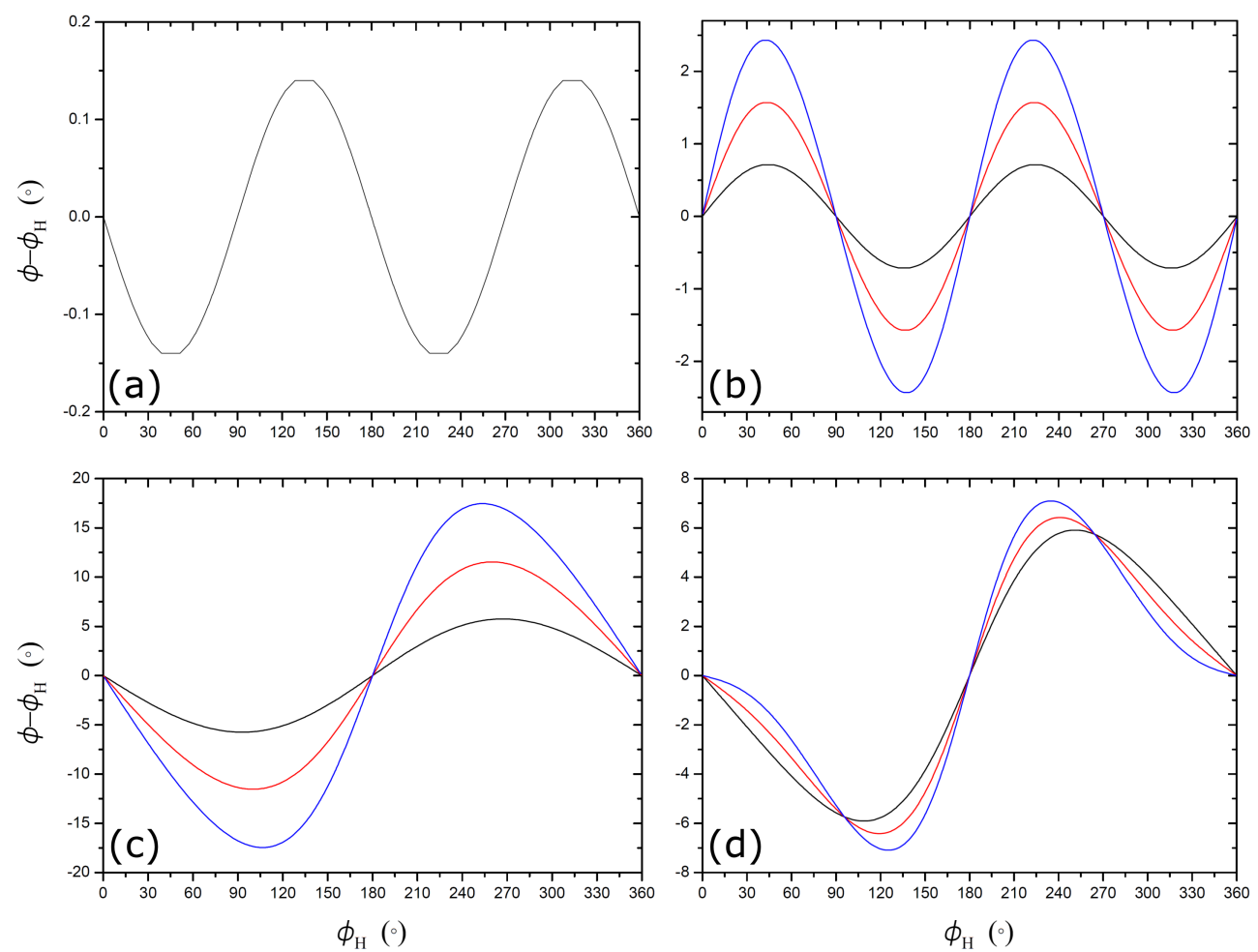

Fig. 3. Dependence on the in-plane angle of the applied magnetic field of the misalignment of the equilibrium direction of magnetization from the direction of the applied magnetic field with different stress anisotropy fields and exchange anisotropy fields. 

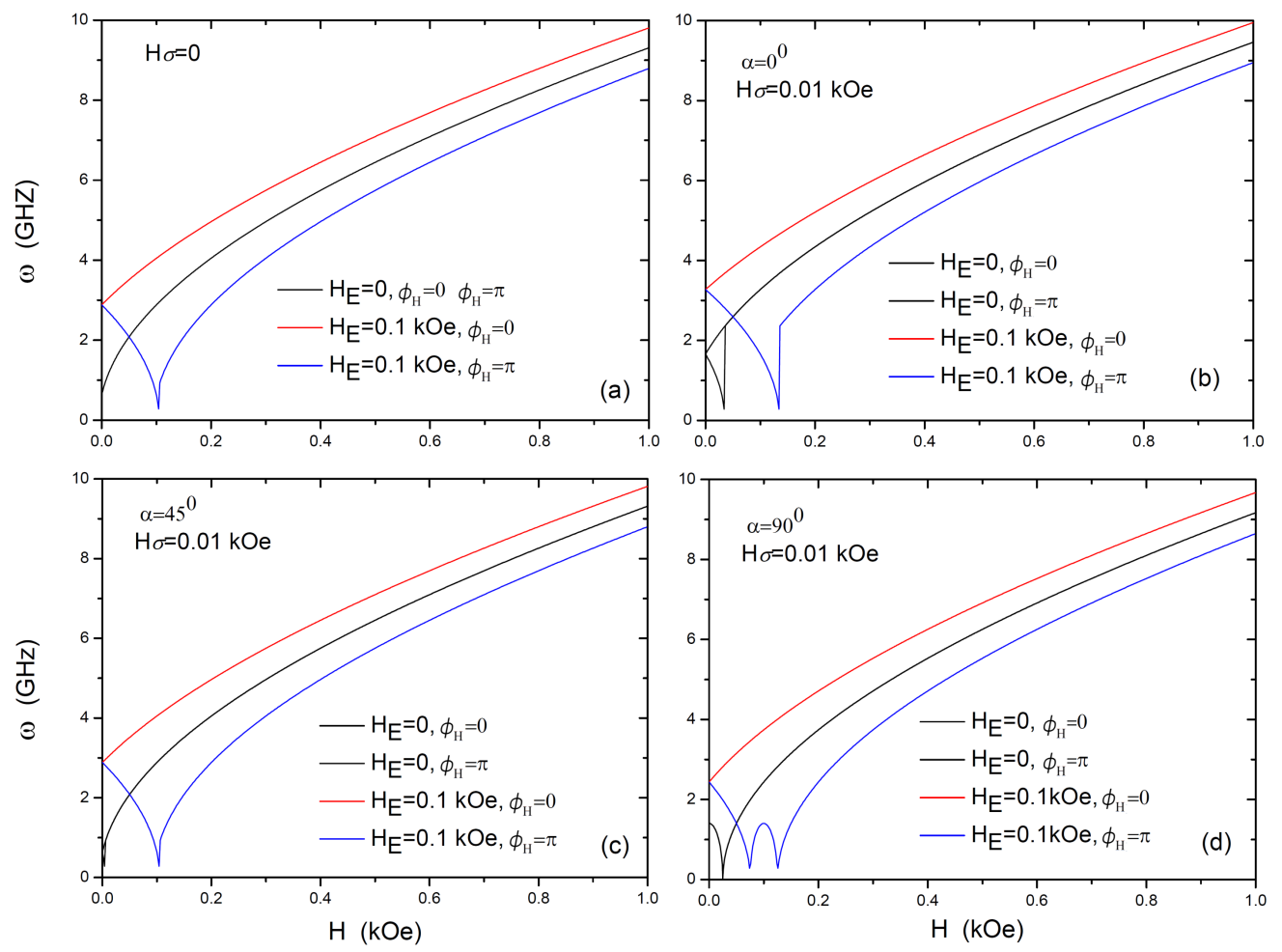

Fig. 4. The resonance frequency as a function of the applied magnetic field with different the stress and the exchange anisotropy fields. Here, $\phi_{H}=0$ (red line) and $\phi_{H}=\pi$ (blue line) for $H_{E}=0.1 \mathrm{kOe}$.

The dominant contribution to the resonance frequency is due to the fact that the stress anisotropy field and exchange anisotropy field change the effective magnetic field of the system.

In addition, the dependence of resonance frequency on the applied magnetic field for different stress anisotropy field directions is calculated and plotted in Fig. 4. When the stress anisotropy field is absent (Fig. 4a) then only two particular cases are considered: the exchange anisotropy equal to zero (the black lines) and $0.1 \mathrm{kOe}$ (the red line for $\phi_{H}=0$ and the blue line for $\left.\phi_{H}=\pi\right)$. We show here that the two black lines overlap. When $H_{E}=0.1 \mathrm{kOe}$, we can see two regions, where the resonance frequency decreases in weak field region and where it increases in strong field region for $\phi_{H}=\pi$, and vanishes at critical field $H_{\text {crit }}=H_{E}+H_{A}$. When the stress anisotropy is included and the direction of stress anisotropy $\alpha=0^{\circ}$ (Fig. 4b), the resonant frequency in $\phi_{H}=0$ and $\phi_{H}=\pi$ are not the same as in weak applied field region in Fig. 4a. Compared with the case without stress anisotropy field, there exists one critical field $H_{\text {crit }}=H_{E}+H_{A}+3 H_{\sigma}$. The critical field depends on the exchange anisotropy field, inplane uniaxial anisotropy field and the stress anisotropy field. This result is different from the report [20], which indicated that the critical field depends on the direction of stress field and the interfacial turning coefficient. In weak field region $H<H_{\text {crit }}$, the resonance frequency is decreased with the applied magnetic field increasing. However, the resonance frequency is jumped at critical field. This is due to the fact that magnetization suddenly rotates from the positive direction of $x$ axis to the negative direction. Besides, the resonance frequency is increased with increase in the applied magnetic field in strong field region $H>H_{\text {crit }}$. The critical field is shifted to high value as exchange anisotropy field is not equal to zero. Furthermore, in Fig. 4(c) we can see that the resonance frequency is the same as the direction of stress anisotropy field along $\alpha=45^{\circ}$ without exchange anisotropy field. From Fig. 4(d), one can distinguish three regions, divided by $H_{1}=H_{E}+H_{A}-3 H_{\sigma}$ and $H_{2}=H_{E}-H_{A}+3 H_{\sigma}$, which are found by minimizing the total energy. (i) region $\mathrm{I}$ : the applied field $H<H_{1}$, the azimuth angle of magnetization $\phi=0^{\circ}$ (the magnetization lies on the $x$ axis); (ii) region II: $H_{1}<H<H_{2}$, the magnetization smoothly rotates from the positive direction of $x$ axis to the negative direction; (iii) region III: $\mathrm{H}>\mathrm{H}_{2}$, where the magnetization is located the direction of applied magnetic field. These conclusions supplement the previous studies [19].

Finally, we plot the angular dependence of the FMR frequency for different directions of stress anisotropy field. Figure 5 shows the evolution of FMR frequency versus the direction of the applied magnetic field that is still applied in the film plane for stress anisotropy field $H_{\sigma}=0.01 \mathrm{kOe}$. In case of no exchange anisotropy (Fig. 5a), the variation of the resonant frequency against applied magnetic field angle is a cosine function. For this case, the easy axis and the hard axis are perpendicular to each other, directed along 
and perpendicular to the stress direction, respectively. When the exchange anisotropy field is increased (Fig. 5b), it leads to a right shift of the lowest resonant frequency as the direction of stress anisotropy field $\alpha$ is changed from $0^{\circ}$ to $90^{\circ}$. In addition, the original symmetrical orientations along the directions $\phi_{H}=0^{\circ}$ and $\phi_{H}=180^{\circ}$ are destroyed.
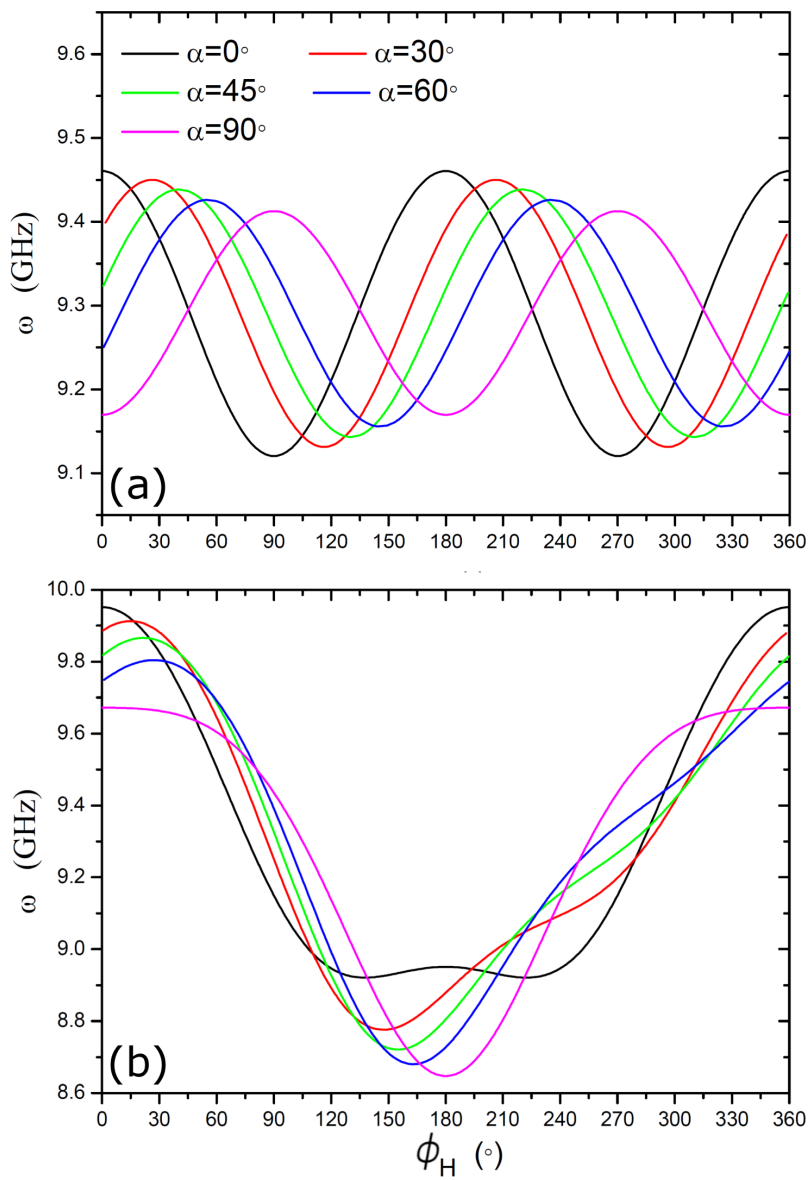

Fig. 5. Resonant frequency versus direction of the applied magnetic field with different angles of the stress anisotropy field: (a) $H_{E}=0$ and (b) $H_{E}=0.1 \mathrm{kOe}$.

\section{Conclusion}

In conclusion, we have derived analytical solution of FMR frequency in ferromagnetic/antiferromagnetic bilayers system with exchange anisotropy, uniaxial magnetocrystalline anisotropy, and stress anisotropy. From the comparison between exchange anisotropy field $H_{E}=0$ and $H_{E}=0.1 \mathrm{kOe}$, we found that the easy axis and the hard axis has a strong field dependence. In case of variation of the applied magnetic field, stress anisotropy field will make resonance frequency shifted to lower value for $\phi_{H}=0^{\circ}$, but we observed that, at low field, there are two easy axes for the direction of applied magnetic field $\phi_{H}=180^{\circ}$ as the stress anisotropy field is along $y$ axis (perpendicular to the direction of exchange anisotropy field). In summary, the controllable stress provides a practical method to engineer integrated microwave devices.

\section{Acknowledgments}

The work has been supported by the National Natural Science Foundation of China under Grant No. 11664030, and by the Science and Technology Research Projects in Colleges and Universities of Inner Mongolia of China under Grant No. NJZY13072, NJZY16014.

\section{References}

[1] W.H. Meiklejohn, C.P. Bean, Phys. Rev. 102, 1413 (1956).

[2] I. Zjutic, J. Fabian, S. Das Sarma, Rev. Mod. Phys. 76, 323 (2004).

[3] S.A. Wolf, D.D. Awschalom, R.A. Buhrman, J.M. Daughton, S.V. Molnar, M.L. Roukes, A.Y. Chtchelkanova, D.M. Treger, Science 294, 1488 (2001).

[4] J. Åkerman, Science 308, 508 (2005).

[5] T. Kimura, M. Hara, Appl. Phys. Lett. 97, 182501 (2010).

[6] S.M. Thompson, J. Phys. D: Appl. Phys. 41, 093001 (2008).

[7] R. Koch, J. Phys.: Condens. Matter6, 9519 (1994).

[8] D. Sander,Rep. Prog. Phys. 62, 809 (1999).

[9] D. Sander, S. Ouazi, A. Enders, Th. GutjahrLoser, V.S. Stepanyuk, D.I. Bazhanov, J. Kirschner, J. Phys.: Condens. Matter 14, 4165 (2002).

[10] D. Sander,Curr. Opin. Solid State Mater. Sci. 7, 51 (2003).

[11] M. Gueye, F. Zighem, M. Belmeguenai, M. Gabor, C. Tiusan, D. Faurie, J. Phys. D: Appl. Phys. 49, 265001 (2016).

[12] A. Layadi, J. Appl. Phys. 87, 1429 (2000).

[13] J.G. Hu, G.J. Jin, Y.Q. Ma, J. Appl. Phys. 91, 2180 (2002).

[14] S.M. Rezende, A. Azevedo, M.A. Lucena, F.M. de Aguiar, Phys. Rev. B 63, 214418 (2002).

[15] B. Khodadadi, J.B. Mohammadi, J.M. Jones, A. Srivastava, C. Mewes, T. Mewes, C. Kaiser, Phys. Rev. Appl. 8, 014024 (2017).

[16] Z. Zhang, P.C. Hammel, M. Midzor, M.L. Roukes, J.R. Childress, Appl. Phys. Lett. 73, 2036 (1998).

[17] A.F. Franco, P. Landeros, J. Phys. D: Appl. Phys. 49, 385003 (2016).

[18] J.B. Mohammadi, J.M. Jones, S. Paul, B. Khodadadi, C.K.A. Mewes, T. Mewes, C. Kaiser, Phys. Rev. B 95, 064414 (2017).

[19] J.H. Rong, G.H. Yun, B. Narsu, D.W.L. Sprung, J. Appl. Phys. 100, 083901 (2006).

[20] J. Pan, J.G. Hu, Phys. Lett. A 358, 236 (2006).

[21] A. Sipeky, A. Ivanyi, Phys. B 372, 177 (2006).

[22] M. Farle, Rep. Prog. Phys. 61, 755 (1998). 
[23] J.H.E. Griffiths, Nature 158, 670 (1946).

[24] E. de Biasi, C.A. Ramos, R.D. Zysler, J. Magn. Magn. Mater. 262, 235 (2003).

[25] D.I. Mircea, T.W. Clinton, Appl. Phys. Lett. 90, 142504 (2007).

[26] C.L. Wang, S.H. Zhang, S.Z. Qiao, H.L. Du, X.M. Liu, R.C. Sun, X.M. Chu, G.X. Miao, Y.Y. Dai, S.S. Kang, S.S. Yan, S.D. Li, Appl. Phys. Lett. 112 192401 (2018).
[27] S.H. Zhang, J.H. Rong, H. Wang, D. Wang, L. Zhang, Surf. Sci. 667, 79 (2018).

[28] S.H. Zhang, J.H. Rong, H. Wang, G.H. Yun, Y.N. Wang, L.B. Bao, J. Magn. Magn. Mater. 461, 23 (2018)

[29] S.H. Zhang, J.H. Rong, H. Wang, D. Wang, L. Zhang, J. Suoercond. Nov. Magn. 31, 1499 (2018).

[30] J. Smit, H.G. Beljers, Philips Res. Rep. 10, 113 (1955). 